\title{
Full discretization of wave equation
}

\author{
Abderrazak Chaoui ${ }^{1 *}$, Fateh Ellaggoune ${ }^{2}$ and Assia Guezane-Lakoud ${ }^{3}$
}

\author{
${ }^{*}$ Correspondence: \\ rchaoui@outlook.fr \\ 'Department of Mathematics, \\ University 8 Mai 1945 Guelma, P.O. \\ Box 401, Guelma, 24000, Algeria \\ Full list of author information is \\ available at the end of the article
}

\begin{abstract}
Rothe's method for time discretization and Crouseix-Raviart nonconforming finite element method to the spatial variable. After introducing error estimators, we prove the equivalence between the error and its indicators.

MSC: Primary 35K55; secondary 35A35

Keywords: Rothe's method; Crouseix-Raviart nonconforming finite element method; wave equation; a posteriori estimate
\end{abstract}

\section{Introduction}

Among commonly used methods for the numerical approach of problems which arise in engineering, for example, Laplace equation and Maxwell system, the finite element method is one of the most relied on methods because it is much more interested in the analysis of the error committed between the exact solution and the approximate solution. In many of these applications, adaptive techniques using a posteriori error estimators have become an indispensable tool. These estimators allow to measure the quality of the computed solution and provide information to control the mesh adaptation algorithm. There are a lot of works on the a posteriori estimators for the elliptic partial differential equations and dynamic partial differential equations. Of these works, it is possible to refer to [1] where the authors considered an elliptic second order boundary value problem approximated by a discontinuous Galerkin method. Time dependent Stokes equations in [2] and second order wave equations in [3] are discretized by Euler's implicit scheme in time and standard finite elements in space. Using Rothe's method in [4] and [5], the authors studied the equation of telegraph and integrodifferential equation with integral conditions (resp.).

The purpose of this work is to combine Rothe's method with nonconforming finite element method of Crouseix-Raviart and to introduce a posteriori error estimators suitable for the wave equation assumptions on the mesh. These indicators can give a good overview of the local distribution of the error and a useful tool for mesh adaptation.

Let $\Omega$ be a bounded open domain of $\mathbb{R}^{d}, d=2$ or 3 with Lipschitz boundary $\Gamma$ that we suppose to be polygonal $(d=2)$ or polyhedral $(d=3)$. We further assume that $\Omega$ is simply connected and that $\Gamma$ is connected. Let $T$ be a fixed positive number,

$$
\begin{aligned}
& \left.\partial_{t t} u-\Delta u=f \quad \text { in } \Omega \times\right] 0, T[, \\
& u(x, 0)=u_{0}(x) \quad \text { in } \Omega \text { at } t=0, \\
& u=0 \quad \text { at } \Gamma \times I, I=(0, T), \\
& \partial_{t} u(x, 0)=u_{1}(x) \quad \text { in } \Omega \text { at } t=0,
\end{aligned}
$$

\section{Springer}

(c) 2015 Chaoui et al. This article is distributed under the terms of the Creative Commons Attribution 4.0 International License (http://creativecommons.org/licenses/by/4.0/), which permits unrestricted use, distribution, and reproduction in any medium, provided you give appropriate credit to the original author(s) and the source, provide a link to the Creative Commons license, and indicate if changes were made. 
where $f \in L^{2}\left((0, T), L^{2}(\Omega)\right), U_{0} \in H_{0}^{1}(\Omega)$ and $U_{1} \in L^{2}(\Omega)$. Under these conditions, problem (1) is equivalent to

$$
\left(\partial_{t t} u, v\right)+(\nabla u, \nabla v)=(f, v), \quad \forall v \in H_{0}^{1}(\Omega) \text { a.e. } t \in(0, T)
$$

has a unique weak solution $C\left((0, T), H_{0}^{1}(\Omega)\right) \cap C^{1}\left((0, T), L^{2}(\Omega)\right)$. If we put $U=\left(\begin{array}{c}u \\ \partial_{t} u\end{array}\right)$ and $F=\left(\begin{array}{l}0 \\ f\end{array}\right)$, then problem (1) can be rewritten as follows:

$$
\left\{\begin{array}{l}
\left.\partial_{t} U-\left(\begin{array}{cc}
0 & 1 \\
\triangle 0
\end{array}\right) U=F \quad \text { in } \Omega \times\right] 0, T[ \\
U=0 \text { on } \Gamma \times I \\
U(\cdot, 0)=U_{0}
\end{array}\right.
$$

with $U_{0}=\left(\begin{array}{l}u_{0} \\ u_{1}\end{array}\right)$.

\section{Time discretization using Rothe's method}

We divide the interval $(0, T)$ into subintervals of length $\tau=\frac{T}{n}$ and denote $u^{j}=u(j \tau, x)$, $x \in \Omega, j=1, \ldots, n$. Successively, for $j=1, \ldots, n$, we solve the linear stationary boundary value problem

$$
\left\{\begin{array}{l}
\frac{u^{j}-2 u^{j-1}+u^{j-2}}{\tau^{2}}-\Delta u^{j}=f^{j} \quad \text { in } \Omega \\
u^{j}=0 \text { on } \Gamma, \\
u^{0}(x, 0)=u_{0} \quad \text { in } \Omega
\end{array}\right.
$$

where $f^{j}=f\left(x, t_{j}\right)=f(x, j \tau)$. Setting $u^{-1}(x)=u_{0}(x)-\tau u_{1}(x)$, define $\delta u^{j}=\frac{u^{j}-u^{j-1}}{\tau}, \delta^{2} u^{j}=$ $\frac{\delta u^{j}-\delta u^{j-1}}{\tau}, j=1, \ldots, n$.

This problem has a unique weak solution $u^{j} \in H_{0}^{1}(\Omega)$ by the Lax-Milgram lemma whose variational formulation is

$$
\left(\delta^{2} u^{j}, v\right)+\left(\nabla u^{j}, \nabla v\right)=\left(f^{j}, v\right), \quad \forall v \in H_{0}^{1}(\Omega)
$$

We define Rothe's functions by a piecewise linear interpolation with respect to the time $t$,

$$
\begin{aligned}
& u^{n}(t, x)=u^{j-1}+\left(t-t_{j-1}\right) \delta u^{j} \quad \text { for } t_{j-1} \leq t \prec t_{j}, j=1, \ldots, n, \\
& \delta u^{n}(t, x)=\delta u^{j-1}+\left(t-t_{j-1}\right) \delta^{2} u^{j} \quad \text { for } t_{j-1} \leq t \prec t_{j}, j=1, \ldots, n,
\end{aligned}
$$

the auxiliary functions are

$$
\begin{aligned}
& \overline{u(t, x)^{n}}= \begin{cases}u^{j} & \text { if } t \in\left(t_{j-1}, t_{j}\right), j=1, \ldots, n, \\
u_{0} & \text { if } t \in[-h, 0],\end{cases} \\
& \overline{\delta u(t, x)^{n}}= \begin{cases}\delta u^{j} & \text { if } t \in\left(t_{j-1}, t_{j}\right), j=1, \ldots, n, \\
u_{1} & \text { if } t \in[-h, 0] .\end{cases}
\end{aligned}
$$

\section{Full discretization}

We consider the following nonconforming finite element method to approximate our problem. For all $j=1, \ldots, n$, we consider a triangulation $\Upsilon_{j h}$ made of triangles $T$ if $d=2$ 
and of tetrahedra if $d=3$ whose edges/faces are denoted by $e$. We assume that this triangulation is regular in Ciarlet's sense ([6], p.124), i.e. , $\exists \sigma \succ 0$ such that $\frac{h_{T}}{\rho_{T}} \leq \sigma, \forall T \in \Upsilon_{j h}$, where $h_{T}$ is the diameter of $T$ and $\rho_{T}$ is the diameter of its largest inscribed bull. We define $h_{j}=\max _{T \in \Upsilon_{j h}} h_{T}$. Let $\zeta_{j h}^{\text {int }}$ be the set of interior edges/faces of $\Upsilon_{j h}$ and $\zeta_{T}$ be the set of edges/faces of the element $T$. For an edge/face $e \in \zeta_{T} \cap \zeta_{K}$, we denote by $h_{e}=\frac{1}{2}\left(\frac{d(T)}{|e|}+\frac{d(K)}{|e|}\right)$ its mean height. Problem (5) is approximated by the Crouseix-Raviart nonconforming finite element space

$$
\begin{aligned}
X_{j h}^{0}= & \left\{v \in L^{2}(\Omega) ; \downarrow \backslash T \in P_{1}, \forall T \in \Upsilon_{j h}, \int_{e} \nu \backslash T=\int_{e} \nu \backslash K, \forall e \in \zeta_{T} \cap \zeta_{K} \cap \zeta_{j h}^{\mathrm{int}},\right. \\
& \left.T, K \in \Upsilon_{j h}, \int_{e} \backslash \backslash T=0, \forall e \in \zeta_{T} \cap \Gamma, T \in \Upsilon_{j h}\right\} .
\end{aligned}
$$

We consider the fully discrete scheme for problem (1): for each $j=1, \ldots, n$, find $u_{h}^{j} \in X_{j h}^{0}$ such that

$$
\tau^{2} \sum_{T \in \Upsilon_{j h}} \int_{T} \nabla u_{h}^{j} \nabla v_{h}=\tau^{2} \int_{\Omega} f^{j} v_{h}-\int_{\Omega}\left(u_{h}^{j}-2 u_{h}^{j-1}+u_{h}^{j-2}\right) v_{h} .
$$

We will use the following Crouseix-Raviart property:

$$
\int_{e}\left[u_{h}\right]_{e}=0, \quad \forall e \in \zeta_{j h}, \forall u_{h} \in X_{j h}^{0}
$$

where the jump of some function $v$ across an edge/face at point $x$ is defined by

$$
[v(x)]_{e}= \begin{cases}\lim _{\alpha \rightarrow 0^{+}} v\left(x+\alpha \eta_{e}\right)-v\left(x-\alpha \eta_{e}\right) & \text { if } e \in \zeta_{j h}^{\mathrm{int}}, \\ v(x) & \text { if } e \in \zeta_{j h} \backslash \zeta_{j h}^{\mathrm{int}},\end{cases}
$$

$\eta_{e}$ denotes the outward normal vector for a boundary edge/face $e$ and $t_{e}=\left(-\eta_{e_{2}}, \eta_{e_{1}}\right)$ is the tangent vector if $\eta_{e}=\left(\eta_{e_{1}}, \eta_{e_{2}}\right)$.

Since $\left[v_{h}\right]$ is linear on $e$, the condition $\int_{e}\left[v_{h}\right]_{e}=0$ is equivalent to the continuity of $v_{h}$ at $e$ barycenter.

For $v_{h} \in X_{j h}^{0}$, we define its broken gradient $\nabla_{h} v$ in $\Omega$ by

$$
\left(\nabla_{h} v\right) \backslash T=\nabla(v \backslash T), \quad \forall T \in \Upsilon_{j h}
$$

We will need local subdomain, also called patches. For any $T \in \Upsilon_{j h}$, let $w_{T}$ be the union of all elements having a common edge/face with $T$. Similarly let $w_{e}$ be the union of all elements having $e$ as an edge/face. Finally, let $w_{x}$ be the union of elements having $x$ as a node, and $\widetilde{w}_{T}$ (resp. $\widetilde{w}_{e}$ ) be the union of all triangles sharing a node with $T$ (resp.e).

Later on, we also need the standard $P_{1}$ conforming finite element spaces:

$$
\begin{aligned}
& V_{j h}=\left\{v \in H^{1}(\Omega) ; v \backslash T \in P_{1}, \forall T \in \Upsilon_{j h}\right\}, \\
& V_{j h}^{0}=V_{j h} \cap H_{0}^{1}(\Omega) .
\end{aligned}
$$


We further need

$$
\begin{aligned}
Y_{j h}= & \left\{v \in L^{2}(\Omega) ; v \backslash T \in H^{1}(\Omega), \forall T \in \Upsilon_{j h}, \int_{e} \nu \backslash T=\int_{e} \nu \backslash K, \forall e \in \zeta_{T} \cap \zeta_{K} \cap \zeta_{j h}^{\mathrm{int}},\right. \\
& \left.T, K \in \Upsilon_{j h}\right\}, \\
Y_{j h}^{0}= & \left\{v \in L^{2}(\Omega) ; v \backslash T \in H^{1}(\Omega), \forall T \in \Upsilon_{j h}, \int_{e} \nu \backslash T=\int_{e} v \backslash K, \forall e \in \zeta_{T} \cap \zeta_{K} \cap \zeta_{j h}^{\mathrm{int}},\right. \\
& \left.T, K \in \Upsilon_{j h}, \int_{e} \nu \backslash T=0, \forall e \in \zeta_{T} \cap \Gamma, T \in \Upsilon_{j h}\right\} .
\end{aligned}
$$

We recall that for a node $x \in N_{j h}$, we denote by $\lambda_{x}$ the standard hat function such that $\lambda_{x}(y)=\delta_{x y}, \forall y \in N_{j h}$, where $N_{j h}$ is the set of nodes of $\Upsilon_{j h}$ and $N_{j h}^{\text {int }}$ denotes the set of interior nodes of $\Upsilon_{j h}$.

Definition 3.1 For $v \in Y_{j h}$ and $w \in Y_{j h}^{0}$, Clément interpolation is defined as follows:

$$
\begin{aligned}
& I_{C} v=\sum_{x \in N_{j h}}\left|w_{x}\right|^{-1}\left(\int_{w_{x}} v\right) \lambda_{x}, \\
& I_{C}^{0} w=\sum_{x \in N_{j h}^{\text {int }}}\left|w_{x}\right|^{-1}\left(\int_{w_{x}} w\right) \lambda_{x} .
\end{aligned}
$$

We define the gradient jump of $u_{h}^{j}$ in normal and tangential direction as follows:

$$
J_{e, \eta}^{j}= \begin{cases}{\left[\nabla u_{h}^{j} \cdot \eta_{e}\right]} & \text { if } e \in \zeta_{j h}^{\text {int }}, \\ 0 & \text { if } e \in \zeta_{j h} \backslash \zeta_{j h}^{\text {int }} .\end{cases}
$$

If $d=2$, then

$$
J_{e, t}^{j}= \begin{cases}{\left[\nabla u_{h}^{j} \cdot t_{e}\right]} & \text { if } e \in \zeta_{j h}^{\mathrm{int}}, \\ -\nabla u_{h}^{j} \cdot t_{e} & \text { if } e \in \zeta_{j h} \backslash \zeta_{j h}^{\text {int }} .\end{cases}
$$

If $d=3$, then

$$
J_{e, t}^{j}= \begin{cases}{\left[\nabla u_{h}^{j} \times n_{e}\right]} & \text { if } e \in \zeta_{j h}^{\mathrm{int}}, \\ -\nabla u_{h}^{j} \times n_{e} & \text { if } e \in \zeta_{j h} \backslash \zeta_{j h}^{\mathrm{int}} .\end{cases}
$$

Lemma 3.1 [7] For all $v \in Y_{j h}$ and $w \in Y_{j h}^{0}$, we have

$$
\begin{aligned}
& \left\|v-I_{C} v\right\|_{T} \lesssim h_{T}\left\|\nabla_{h} v\right\|_{\widetilde{w}_{T}}, \quad \forall T \in \Upsilon_{j h}, \\
& \left\|v-I_{C} v\right\|_{e} \lesssim h_{e}^{\frac{1}{2}}\left\|\nabla_{h} v\right\|_{\widetilde{w}_{e}}, \quad \forall e \in \zeta_{j h}, \\
& \left\|w-I_{C}^{0} w\right\|_{T} \lesssim h_{T}\left\|\nabla_{h} w\right\|_{\widetilde{w}_{T}}, \quad \forall T \in \Upsilon_{j h}, \\
& \left\|w-I_{C}^{0} w\right\|_{e} \lesssim h_{e}^{\frac{1}{2}}\left\|\nabla_{h} w\right\|_{\widetilde{w}_{e}}, \quad \forall e \in \zeta_{j h}^{\mathrm{int}}, \\
& \left\|\nabla I_{C}^{0} w\right\| \lesssim\left\|\nabla_{h} w\right\|_{\widetilde{w}_{T}}, \quad \forall T \in \Upsilon_{j h} .
\end{aligned}
$$


Next, we need the following Green's formulas.

If $D$ is open bounded of $\mathbb{R}^{2}$ and $v, w \in H^{1}(D)$, then

$$
\int_{D} \nabla v \operatorname{curl} w=\int_{\partial D} v \operatorname{curl} w \cdot \eta=\int_{\partial D} \nabla v \cdot t w
$$

where $t$ is the unit tangent vector along $\partial D$ and $\operatorname{curl} w=\left(\begin{array}{c}\partial_{2} w \\ -\partial_{1} w\end{array}\right)$.

Similarly if $D$ is open bounded of $\mathbb{R}^{3}$ and $v \in H^{1}(D), w \in H^{1}(D)^{3}$, then we have

$$
\int_{D} \nabla v \operatorname{curl} w=\int_{\partial D} v \operatorname{curl} w \cdot \eta=\int_{\partial D}(\nabla v \times \eta) \cdot w
$$

\section{A posteriori analysis of time discretization}

For each $j, j=1, \ldots, n$, the refinement indicator is defined by

$$
\eta_{t}^{j}=\tau\left\|\nabla_{h}\left(u_{h}^{j+1}-u_{h}^{j}\right)\right\|+\tau\left\|\frac{u_{h}^{j+1}-2 u_{h}^{j}+u_{h}^{j-1}}{\tau}\right\|,
$$

$e^{\tau}=u-u^{n}$ indicate the error with respect to the discretization time.

Proposition 4.1 (Upper and lower bounds of the error in time) The following a posteriori error estimate holds for all $t_{j+1}, j=1, \ldots, n-1$ :

$$
\begin{aligned}
& \left\|\left(\partial_{t} u\right)\left(t_{j+1}\right)-\frac{u^{j+1}-u^{j}}{\tau}\right\|_{H^{-1}(\Omega)}+\left\|u\left(t_{j+1}\right)-u^{j+1}\right\| \\
& \lesssim \sum_{m=1}^{j} \eta_{t}^{m}+\tau\left\|\nabla U_{0}\right\|+\tau^{2}\left\|\nabla U_{1}\right\|+\sum_{m=1}^{j} \tau \sum_{k=0}^{1}\left\|\nabla\left(u^{m+1-k}-u_{h}^{m+1-k}\right)\right\| \\
& +\sum_{m=1}^{j} \tau \sum_{k=0}^{1}\left\|\delta u^{m+1-k}-\delta_{h}^{m+1-k}\right\| \\
& \eta_{t}^{j} \leq \sum_{k=0}^{1}\left\|\left(\partial_{t} u\right)\left(t_{j+1-k}\right)-\delta u^{j+1-k}\right\|_{H^{-1}(\Omega)}+\left\|u\left(t_{j+1}\right)-\overline{w^{j+1-k}}\right\|+\left\|\int_{t_{j}}^{t j+1} \nabla\left(u-u^{n}\right)(s) d s\right\| \\
& +\left\|\int_{t_{j}}^{t j+1}\left(\partial_{t} u-\delta u^{n}\right)(s) d s\right\|+\tau \sum_{k=0}^{1}\left\|\nabla\left(u^{j+1-k}-u_{h}^{j+1-k}\right)\right\| \\
& +\tau \sum_{k=0}^{1}\left\|\delta u^{j+1-k}-\delta_{h} u_{h}^{j+1-k}\right\|
\end{aligned}
$$

Proof See [3].

\section{A posteriori analysis of space discretization}

The error indicator is defined by

$$
\eta_{T}^{j}=h_{T}\left\|f_{h}^{j}-\frac{u_{h}^{j}-2 u_{h}^{j-1}+u_{h}^{j-2}}{\tau^{2}}\right\|+\sum_{e \in \zeta_{j h}} h_{e}^{\frac{1}{2}}\left(\left\|J_{e, \eta}^{j}\right\|_{e}+\left\|J_{e, t}^{j}\right\|_{e}\right),
$$


the global error estimator $\eta^{j}$ is given by

$$
\eta^{j}=\sqrt{\sum_{T \in \Upsilon_{j h}}\left(\eta_{T}^{j}\right)^{2}},
$$

the higher order term depending on the datum $f$ is defined as

$$
\operatorname{osc}\left(f, w_{T}\right)^{2}=\sum_{T \in \Upsilon_{j h}} h_{T}^{2}\left\|f-f_{h}^{j}\right\|_{w_{T}}^{2}, \quad \text { where }\left(f_{h}^{j}\right) \backslash T:=\frac{1}{|T|} \int_{T} f_{h}^{j}, T \in \Upsilon_{j h} .
$$

Our main result is the following theorem.

Theorem 5.1 (Upper bound) The following inequality holds:

$$
\begin{aligned}
\left\|e^{n}\right\|^{2}+\sum_{j=1}^{n} \tau^{2}\left\|\nabla_{h} e^{j}\right\|^{2} \lesssim & \left\|e^{0}\right\|^{2}+\left\|e^{1}\right\|^{2}+\tau^{2} \sum_{j=1}^{n} \operatorname{osc}\left(f, w_{T}\right)^{2} \\
& +\sum_{j=1}^{n} \max \left\{h_{j}^{2}, \tau^{2}\right\}\left(\eta^{j}\right)^{2} .
\end{aligned}
$$

To prove this theorem we need some lemmas. As our approximated scheme is a nonconforming one, we need to use an appropriate Helmholtz decomposition of the error.

Lemma 5.1 (Helmholtz decomposition of the error) Let $e^{j}=u^{j}-u_{h}^{j}$, then we have the following decomposition of error $e^{j}$ :

$$
\nabla_{h} e^{j}=\nabla \varphi^{j}+\operatorname{curl} \chi^{j}
$$

with $\chi^{j} \in H^{1}(\Omega)$ and $\varphi^{j} \in H_{0}^{1}(\Omega)$; furthermore, the following inequalities hold:

$$
\begin{aligned}
& \left\|\nabla \varphi^{j}\right\| \leq\left\|\nabla_{h} e^{j}\right\|, \\
& \left\|\operatorname{curl} \chi^{j}\right\| \leq\left\|\nabla_{h} e^{j}\right\| .
\end{aligned}
$$

Proof We consider the following problem: find $\varphi^{j} \in H_{0}^{1}(\Omega)$, a solution of

$$
\left\{\begin{array}{l}
\operatorname{div}\left(\nabla_{h} e^{j}-\nabla \varphi^{j}\right)=0 \quad \text { in } \Omega, \\
\varphi^{j=0} \text { on } \Gamma .
\end{array}\right.
$$

The weak formulation of that problem (29) is

$$
\int_{\Omega} \nabla \varphi^{j} \nabla v=\int_{\Omega} \nabla_{h} e^{j} \nabla v, \quad \forall v \in H_{0}^{1}(\Omega) .
$$

As the vector field $\left(\nabla_{h} e^{j}-\nabla \varphi^{j}\right)$ is divergence-free in $\Omega$, i.e., $\operatorname{div}\left(\nabla_{h} e^{j}-\nabla \varphi^{j}\right)=0$ in $\Omega$, by Theorem 1.3.1 of [8] if $d=2$ or Theorem 1.3.4 of [8] if $d=3$, there exists $\chi^{j} \in H^{1}(\Omega)$ if $d=2$ and $\chi^{j} \in H^{1}(\Omega)^{3}$ if $d=3$ such that

$$
\operatorname{curl} \chi^{j}=\nabla_{h} e^{j}-\nabla \varphi^{j}
$$


Estimate (27) directly follows by taking $v=\varphi^{j}$ in (30). To prove the inequality, we use identity (26) and we get

$$
\int_{\Omega}\left|\operatorname{curl} \chi^{j}\right|^{2}=\int_{\Omega} \operatorname{curl} \chi^{j}\left(\nabla_{h} e^{j}-\nabla \varphi^{j}\right) .
$$

Using Green's formula and taking into account that $\varphi^{j}=0$ on $\Gamma$, we get

$$
\int_{\Omega}\left|\operatorname{curl} \chi^{j}\right|^{2}=\int_{\Omega} \operatorname{curl} \chi^{j} \nabla_{h} e^{j}
$$

The Cauchy-Schwarz inequality implies

$$
\left\|\operatorname{curl} \chi^{j}\right\| \leq\left\|\nabla_{h} e^{j}\right\|
$$

Lemma 5.2 The error satisfies the following identity:

$$
\sum_{T \in \Upsilon_{j h}} \int_{T} \nabla_{h} e^{j} \nabla v_{h}=\int_{\Omega} \frac{-e^{j}+2 e^{j-1}-e^{j-2}}{\tau^{2}} v_{h}, \quad \forall v_{h} \in V_{j h}^{0}(\Omega) .
$$

Proof We only need to take $v=v_{h}$ in (5), then taking the difference between (5) and (10) we get the result.

Lemma 5.3 Let $\varphi_{h} \in V_{\text {jh }}$ if $d=2$ and $\varphi_{h} \in\left(V_{j h}\right)^{3}$ if $d=3$, then the error verifies

$$
\sum_{T \in \Upsilon_{j h}} \int_{T} \nabla_{h} e^{j} \operatorname{curl} \varphi_{h}=0
$$

Proof We integrate by parts the expression $\int_{\Omega} \nabla_{h} e^{j} \operatorname{curl} \varphi_{h}$, using Green's formula and taking into account that $u^{j} \in H_{0}^{1}(\Omega)$, then we use the property of finite elements of CrouseixRaviart $\left(\int_{e}\left[u_{h}^{j}\right]=0\right)$ and get (33).

Lemma 5.4 Let $\varphi \in H^{1}(\Omega)$ if $d=2$ and $\varphi \in\left(H^{1}(\Omega)\right)^{3}$ if $d=3$, then we have

$$
\int_{\Omega} \nabla_{h} e^{j} \operatorname{curl} \varphi=\sum_{e \in \zeta_{j h}} \int_{E} J_{E, t}^{j} \cdot \varphi
$$

Proof The integration by parts and Green's formula give us

$$
\int_{\Omega} \nabla_{h} e^{j} \operatorname{curl} \varphi=\int_{\Omega} \nabla u^{j} \operatorname{curl} \varphi-\sum_{T \in \Upsilon_{j h}} \int_{T} \nabla u_{h}^{j} \operatorname{curl} \varphi_{h}=-\sum_{T \in \Upsilon_{j h}} \int_{\partial T} \nabla u_{h}^{j} \cdot t_{T} \varphi,
$$

because $u^{j} \in H_{0}^{1}(\Omega)$, and according to the definition of $J_{E, t}^{j}$ we find (34).

Lemma 5.5 Let $\varphi \in H_{0}^{1}(\Omega)$, then $e^{j}$ verifies

$$
\sum_{T \in \Upsilon_{j h}} \int_{T} \nabla_{h} e^{j} \nabla \varphi=\sum_{T \in \Upsilon_{j h}} \int_{T}\left(f^{j}-\frac{u^{j}-2 u^{j-1}+u^{j-2}}{\tau^{2}}\right) \varphi+\sum_{e \in \zeta j h} \int_{e} J_{e, \eta}^{j} \cdot \varphi .
$$


Proof We integrate by parts the expression $\sum_{T \in \Upsilon_{j h}} \int_{T} \nabla_{h} e^{j} \nabla \varphi$ with $\Delta u=0$ on each element $T \in \Upsilon_{j h}$, and from the definition of $J_{e, \eta}^{j}$ we conclude the proof.

Remark 5.1 Lemmas 5.4, 5.5 imply that $\forall \varphi \in H_{0}^{1}(\Omega)$ and $\chi \in H^{1}(\Omega)$ if $d=2$ and $\chi \in$ $\left(H^{1}(\Omega)\right)^{3}$ if $d=3$, we have

$$
\begin{aligned}
\sum_{T \in \Upsilon_{j h}} \int_{T} \nabla_{h} e^{j}(\nabla \varphi+\operatorname{curl} \chi)= & \sum_{T \in \Upsilon_{j h}} \int_{T}\left(f^{j}-\frac{u^{j}-2 u^{j-1}+u^{j-2}}{\tau^{2}}\right) \varphi \\
& +\sum_{e \in \zeta_{j h}} \int_{e}\left(J_{e, \eta}^{j} \cdot \varphi+J_{\eta, t}^{j} \cdot \chi\right) .
\end{aligned}
$$

Note that the local error estimator $\eta_{T}^{j}$ is inspired by the latter identity.

Proof From what precedes (Lemmas 5.2 and 5.5), we can easily prove that

$$
\begin{aligned}
\tau^{2} \int_{\Omega} \nabla_{h} e^{j} \nabla \varphi^{j}= & \tau^{2} \int_{\Omega}\left(f^{j}-\frac{u^{j}-2 u^{j-1}+u^{j-2}}{\tau^{2}}\right)\left(\varphi^{j}-I_{C}^{0} \varphi^{j}\right) \\
& +\tau^{2} \sum_{e \in \zeta_{j h}} \int_{E} J_{e, \eta}^{j}\left(\varphi^{j}-I_{C}^{0} \varphi^{j}\right)-\int_{\Omega}\left(e^{j}-2 e^{j-1}+e^{j-2}\right) \varphi^{j} .
\end{aligned}
$$

From Lemmas 5.3 and 5.4 we get

$$
\int_{\Omega} \nabla_{h} e^{j} \operatorname{curl} \chi^{j}=\sum_{E \in \zeta_{j h}} \int_{E} J_{E, t}^{j}\left(\chi^{j}-I_{C} \chi^{j}\right) .
$$

By using the Helmholtz decomposition of the error and identities (35)-(36), we obtain

$$
\begin{aligned}
\left\|e^{j}\right\|+\tau^{2} \int_{\Omega}\left|\nabla_{h} e^{j}\right|^{2}= & \tau^{2} \int_{\Omega} \nabla_{h} e^{j} \nabla I_{C}^{0}\left(e^{j}-\varphi^{j}\right)+\left(-e^{j}+2 e^{j-1}-e^{j-2}, e^{j}-\varphi^{j}-I_{C}^{0}\left(e^{j}-\varphi^{j}\right)\right) \\
& +\tau^{2} \int_{\Omega}\left(f^{j}-\frac{u^{j}-2 u^{j-1}+u^{j-2}}{\tau^{2}}\right)\left(\varphi^{j}-I_{C}^{0} \varphi^{j}\right)+\left(2 e^{j-1}-e^{j-2}, e^{j}\right) \\
& +\tau^{2} \sum_{e \in \zeta_{j h}} \int_{e}\left(J_{e, \eta}^{j}\left(\varphi^{j}-I_{C}^{0} \varphi^{j}\right)+J_{e, t}^{j}\left(\chi^{j}-I_{C} \chi^{j}\right)\right) .
\end{aligned}
$$

The Cauchy-Schwarz inequality and estimate (19) give

$$
\begin{aligned}
\sum_{e \in \zeta_{j h}} \int_{e} J_{e, \eta}^{j}\left(\varphi^{j}-I_{C} \varphi^{j}\right) & \leq \sum_{e \in \zeta_{j h}}\left\|J_{e, \eta}^{j}\right\|\left\|\varphi^{j}-I_{C} \varphi^{j}\right\|_{e} \lesssim \sum_{e \in \zeta_{j h}}\left\|J_{e, \eta}^{j}\right\| h_{e}^{\frac{1}{2}}\left\|\nabla \varphi^{j}\right\|_{\widetilde{w}_{e}} \\
& \lesssim \sum_{e \in \zeta_{j h}} \eta_{T}^{j}\left\|\nabla \varphi^{j}\right\|_{\widetilde{w}_{e}} \lesssim \eta^{j}\left\|\nabla \varphi^{j}\right\| .
\end{aligned}
$$

Similarly, using the Cauchy-Schwarz inequality and estimate (17), we get

$$
\sum_{e \in \zeta_{j h}} \int_{E} J_{E, t}^{j}\left(\chi^{j}-I_{C} \chi^{j}\right) \lesssim \eta^{j}\left\|\nabla \chi^{j}\right\| .
$$


By using the Helmholtz decomposition, Green's formula and identity (36), we find

$$
\begin{aligned}
\int_{\Omega}\left|\operatorname{curl} \chi^{j}\right|^{2} & =\int_{\Omega}\left(\nabla_{h} e^{j}-\nabla_{h} \varphi^{j}\right) \operatorname{curl} \chi^{j}=\int_{\Omega} \nabla_{h} e^{j} \operatorname{curl} \chi^{j} \\
& =\sum_{e \in \zeta_{j h}} \int_{e} J_{e, t}^{j}\left(\chi^{j}-I_{C} \chi^{j}\right) \lesssim \eta^{j}\left\|\nabla \chi^{j}\right\| .
\end{aligned}
$$

The Cauchy-Schwarz inequality and estimate (17) imply

$$
\int_{\Omega}\left|\operatorname{curl} \chi^{j}\right|^{2} \lesssim \eta^{j}\left\|\nabla \chi^{j}\right\|
$$

Knowing that

$$
\left\|\operatorname{curl} \chi^{j}\right\|=\left\|\nabla \chi^{j}\right\|
$$

we get

$$
\left\|\nabla \chi^{j}\right\| \lesssim \eta^{j}
$$

and consequently

$$
\sum_{e \in \zeta_{j h}} \int_{e} J_{e, t}^{j}\left(\chi^{j}-I_{C} \chi^{j}\right) \lesssim\left(\eta^{j}\right)^{2}
$$

On the other hand, the Cauchy-Schwarz inequality and estimate (18) give

$$
\begin{aligned}
\sum_{T \in \Upsilon_{j h}} \int_{T}\left(f^{j}-f_{h}^{j}\right)\left(\varphi^{j}-I_{C}^{0} \varphi^{j}\right) & \lesssim \sum_{T \in \Upsilon_{j h}} h_{T}\left\|f^{j}-f_{h}^{j}\right\|\left\|\nabla \varphi^{j}\right\|_{\widetilde{w}_{T}} \\
& \lesssim \operatorname{osc}\left(f_{\widetilde{w}_{T}}\right)\|\nabla \varphi\| .
\end{aligned}
$$

Similarly, we have

$$
\left|\left(-e^{j}+2 e^{j-1}-e^{j-2}, \varphi^{j}-e^{j}-I_{C}^{0}\left(\varphi^{j}-e^{j}\right)\right)\right| \lesssim h_{T}\left\|e^{j}-2 e^{j-1}+e^{j-2}\right\|\left\|\nabla\left(e^{j}-\varphi^{j}\right)\right\| .
$$

Proceeding as in (40) we can prove that

$$
\left\|\nabla\left(e^{j}-\varphi^{j}\right)\right\| \lesssim \eta^{j}
$$

which implies that

$$
\left|\left(-e^{j}+2 e^{j-1}-e^{j-2}, \varphi^{j}-e^{j}-I_{C}^{0}\left(\varphi^{j}-e^{j}\right)\right)\right| \lesssim h_{T} \eta^{j}\left\|e^{j}-2 e^{j-1}+e^{j-2}\right\| .
$$

To estimate $\left\|\nabla \varphi^{j}\right\|$, we have

$$
\left\|\nabla \varphi^{j}\right\| \leq\left\|\nabla_{h}\left(\varphi^{j}-e^{j}\right)\right\|+\left\|\nabla_{h} e^{j}\right\| \leq C\left(\eta^{j}\right)^{2}+\left\|\nabla_{h} e^{j}\right\|^{2} .
$$


For the residual element, using the Cauchy-Schwarz inequality and estimation (18), we get

$$
\begin{aligned}
\sum_{T \in \Upsilon_{j h}} \int_{T}\left(f_{h}^{j}-\frac{u^{j}-2 u^{j-1}+u^{j-2}}{\tau^{2}}\right)\left(\varphi^{j}-I_{C}^{0} \varphi^{j}\right) & \lesssim \sum_{T \in \Upsilon_{j h}} h_{T}\left\|f_{h}^{j}-\frac{u^{j}-2 u^{j-1}+u^{j-2}}{\tau^{2}}\right\|\left\|\nabla \varphi^{j}\right\|_{\widetilde{w}_{T}} \\
& \lesssim \eta^{j}\left\|\nabla \varphi^{j}\right\| .
\end{aligned}
$$

Using the $\epsilon$-inequality and replacing the previous estimates in (37), we find

$$
\left\|e^{j}\right\|^{2}+\tau^{2} \int_{\Omega}\left|\nabla_{h} e^{j}\right|^{2} \leq \frac{1}{2}\left\|e^{j-1}\right\|^{2}+\frac{1}{2}\left\|e^{j-2}\right\|^{2}+C\left(\max \left\{h_{j}^{2}, \tau^{2}\right\}\left(\eta^{j}\right)^{2}+\tau^{2} \cdot \operatorname{osc}\left(f_{\widetilde{w}_{T}}\right)^{2}\right) .
$$

Summing from $j=2$ until $n$ results in

$$
\begin{aligned}
& \left\|e^{n}\right\|^{2}+\sum_{j=1}^{n} \tau^{2} \int_{\Omega}\left|\nabla_{h} e^{j}\right|^{2} \lesssim\left\|e^{0}\right\|^{2}+\left\|e^{1}\right\|^{2}+\sum_{j=1}^{n} \max \left\{h_{j}^{2}, \tau^{2}\right\}\left(\eta^{j}\right)^{2} \\
& +\sum_{j=1}^{n} \tau^{2} \cdot \operatorname{osc}\left(f_{, \widetilde{w}_{T}}\right)^{2}
\end{aligned}
$$

Theorem 5.2 (Lower bound of the error) $[9,10]$ For each element $T \in \Upsilon_{j h}, j=2, \ldots, n$, the following estimate holds:

$$
\eta_{T}^{j} \lesssim h_{T}\left\|\frac{u_{h}^{j}-2 u_{h}^{j-1}+u^{j-2}}{\tau^{2}}\right\|+\left\|\nabla_{h} e^{j}\right\|_{w_{T}}+h_{T}\left\|f^{j}-f_{h}^{j}\right\|_{w_{T}} .
$$

\section{Competing interests}

The authors declare that they have no competing interests.

\section{Authors' contributions}

All authors read and approved the final manuscript.

\section{Author details}

${ }^{1}$ Department of Mathematics, University 8 Mai 1945 Guelma, P.O. Box 401, Guelma, 24000, Algeria. ${ }^{2}$ Laboratory of Applied Mathematics and Modeling, University 8 Mai 1945 Guelma, P.O. Box 401, Guelma, 24000, Algeria. ${ }^{3}$ Advanced Materials Laboratory, University of Badji Mokhtar Annaba, P.O. Box 12, Annaba, 23000, Algeria.

\section{Acknowledgements}

The authors are grateful for the referees' careful reading and comments on this paper that led to the improvement of the original manuscript. This work is supported by the MESRS of Algeria (CNEPRU Project B01520130004).

Received: 14 April 2015 Accepted: 19 July 2015 Published online: 07 August 2015

\section{References}

1. Cochez-Dhondt, S, Nicaise, S: Equilibrated error estimators for discontinuous Galerkin methods. Numer. Methods Partial Differ. Equ. 24, 1236-1252 (2008)

2. Bernardi, C, Verfürth, R: A posteriori error analysis of the fully discretized time-dependent Stokes equations. M2AN Math. Model. Numer. Anal. 38, 437-455 (2004)

3. Bernardi, C, Süli, E: Time and space adaptivity for the second-order wave equation. Math. Models Methods Appl. Sci. 15(2), 199-225 (2005)

4. Guezane-Lakoud, A, Belakroum, D: Rothe's method for a telegraph equation with integral conditions. Nonlinear Anal. 70, 3842-3853 (2009)

5. Guezane-Lakoud, A, Jasmati, MS, Chaoui, A: Rothe's method for an integrodifferential equation with integral conditions. Nonlinear Anal. 72, 1522-1530 (2010)

6. Ciarlet, PG: The Finite Element Method for Elliptic Problems. Studies in Mathematics and Its Applications, vol. 4. North-Holland, Amsterdam (1978)

7. Clément, P: Approximation by finite element functions using local regularization. Rev. Fr. Autom. Inform. Rech. Opér., Anal. Numér. 9(R-2), 77-84 (1975) 
8. Girault, V, Raviart, PA: Finite Element Methods for Navier-Stokes Equations: Theory and Algorithms. Springer Series in Computational Mathematics, vol. 5. Springer, Berlin (1986)

9. Dari, E, Duran, R, Padra, C, Vampa, V: A posteriori error estimators for nonconforming finite element methods. Modél. Math. Anal. Numér. 30(4), 385-400 (1996)

10. Nicaise, $\mathrm{S}$, Soualem, $\mathrm{N}$ : A posteriori error estimates for a nonconforming finite element discretization of the heat equation. M2AN Math. Model. Numer. Anal. 39(2), 319-348 (2005)

Submit your manuscript to a SpringerOpen ${ }^{\circ}$ journal and benefit from:

- Convenient online submission

- Rigorous peer review

- Immediate publication on acceptance

- Open access: articles freely available online

- High visibility within the field

Retaining the copyright to your article

Submit your next manuscript at $\gg$ springeropen.com 